\title{
Tracer experiments in periodical heterogeneous model porous medium
}

\author{
Samer Majdalani ${ }^{1, \star}$, Carole Delenne ${ }^{1,2, \star \star}$, and Vincent Guinot ${ }^{1,2, \star \star \star}$ \\ ${ }^{1}$ Univ. Montpellier, HSM (UMR 5569, CNRS, IRD, UM), CC0057, 163 rue Auguste Broussonnet 34090 Montpellier. \\ ${ }^{2}$ Inria Sophia, Team Lemon
}

\begin{abstract}
It is established that solute transport in homogenous porous media follows a classical ' $S$ ' shape breakthrough curve that can easily be modelled by a convection dispersion equation. In this study, we designed a Model Heterogeneous Porous Medium (MHPM) with a high degree of heterogeneity, in which the breakthrough curve does not follow the classical ' $S$ ' shape. The contrast in porosity is obtained by placing a cylindrical cavity ( $100 \%$ porosity) inside a $40 \%$ porosity medium composed with $1 \mathrm{~mm}$ glass beads. Step tracing experiments are done by injecting salty water in the study column initially containing deionised water, until the outlet concentration stabilises to the input one. Several replicates of the experiment were conducted for $n=1$ to 6 MHPM placed in series. The total of 116 experiments gives a high-quality database allowing the assessment of experimental uncertainty. The experimental results show that the breakthrough curve is very different from the 'S' shape for small values of $n$, but the more $n$ increases, the more the classical shape is recovered.
\end{abstract}

\section{Introduction}

To predict the fate of contaminants in natural soils, it is important to understand solute transport in porous media. What characterizes natural soils is their high degree of heterogeneity, which impacts solute transport mechanisms. The classical Advection-Dispersion (AD) model is generally used to describe solute transport in porous media, and it is characterized by a typical ' $S$ ' shape breakthrough curve. The AD model is capable of reproducing tracer experiments in homogeneous porous media, but it fails to reproduce tracer experiments in heterogeneous porous media because breakthrough curves do not have any more the typical 'S' shape. In the literature, many laboratory tracer experiments in porous media can be found [1] but they are generally related to homogeneous porous media. Therefore, laboratory experiments on heterogeneous porous media are very few in comparison to those done on homogeneous porous media (see e.g. [2-6]).

In order to understand solute transport mechanisms, we use laboratory tracer experiments on Model Heterogeneous Porous Media (MHPM), built with a high degree of heterogeneity to guarantee that the effects of material heterogeneity are clearly visible. Moreover, we study the effect of periodicity of MHPM on breakthrough curves (and thus on solute transport behaviour) by assembling several MHPM in series.

Our experimental curves are characterized by a very fine sampling (one data every 5 seconds), as well as a high number of replicates in order to eliminate statistical errors

\footnotetext{
^e-mail: samer.majdalani@umontpellier.fr

$\star \star$ e-mail: carole.delenne@umontpellier.fr

$\star \star \star$ e-mail: vincent.guinot@umontpellier.fr
}

in breakthrough curves. We can resume the features of our study by the following characteristics: $i$ ) high quality tracer experiments, $i i$ ) high degree of heterogeneity of the porous media, and iii) effect of periodicity of the heterogeneous porous media on solute transport.

In the following, experimental setup is detailed in section 2 and results are given in section 3 in terms of experiment and comparison with the classical advectiondispersion model. Section 4 provides conclusions about this experimental work.

\section{Experimental setup}

Each Model Heterogeneous Porous Medium (MHPM) is built in a PVC column of $10 \mathrm{~cm}$ in diameter, $15 \mathrm{~cm}$ in length. As shown in Figure 1, it consists of a cylindrical cavity with $100 \%$ porosity placed in the centre of the column and surrounded by a medium composed of $1 \mathrm{~mm}$ glass beads ( $40 \%$ porosity). Four inlets and outlets occupy the centre of gravity and the three vertices of an equilateral triangle. The centre of gravity is on the central axis of the column and the three vertices are $1 \mathrm{~cm}$ away from the column periphery. The internal cavity is a tube that is punched on top and bottom and covered with a $500 \mu \mathrm{m}$ sieve. The sieve impedes the glass spheres from entering inside the cavity and guaranties that only water (and solute) can transfer through it. A similar sieve is also used to prevent glass spheres from clogging the inlets and outlets, possibly blocking the flow.

The MHPM initially contains deionised water. The tracer used in the experiments is composed of deionised water supplemented with $\mathrm{NaCl}$ at $C_{0}=0.1 \mathrm{Mol} / \mathrm{l}$. A constant discharge $(7.5 \mathrm{l} / \mathrm{h})$ of this salty water is prescribed at 

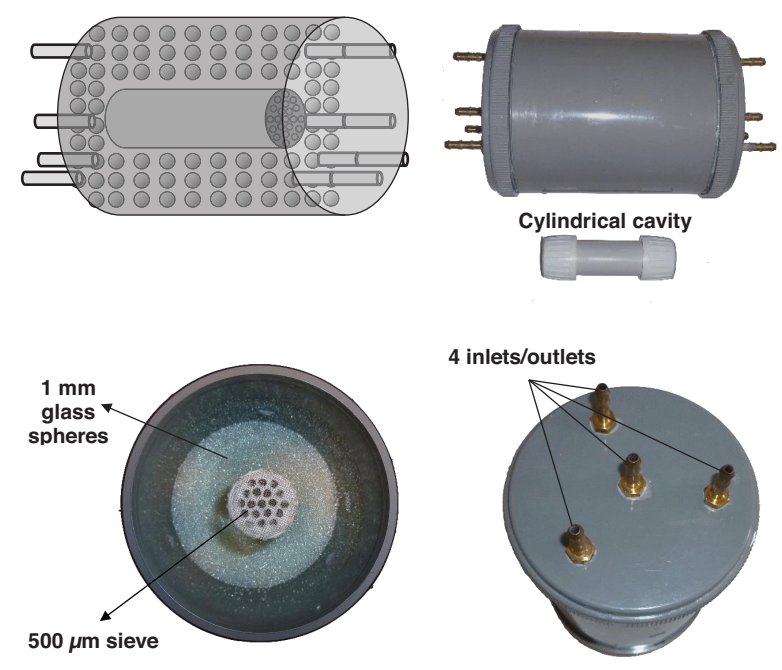

Figure 1. A Model Heterogeneous Porous Medium (MHPM).

the upstream boundary by a peristaltic pump (Gilson MP3) and is equally distributed between the four inlets.

The tracer exits the column through the four outlets that converge in a unique one after which the concentration is measured using a conductimeter (WTW TetraCon 325). Measurements are saved on a data logger (Campbell CR1000) every $5 \mathrm{~s}$ until the outlet concentration $C(t)$ stabilizes to $C_{0}(t)$. The duration $T_{\max }$ for one MHPM of $15 \mathrm{~cm}$ length is about 15 minutes.

To study the periodical effect on solute transport in heterogeneous media, 12 of the MHPM presented in [7] were used and experiments were conducted with $n=1$ to 6 MHPM placed in series. An important difference with [7] stems in the in-between MHPM connection. Indeed, after each MHPM a convergence flow outlet is followed by a divergence inlet for the next MHPM. The period of the periodic heterogeneous porous media is thus defined by one MHPM. Figure 2 represents the general sketch of the experimental setup with an example of a series of $n=4$ MHPM. At the end of the setup, the cumulative outlet volume $V$ is measured by weighting the effluent every $5 \mathrm{~s}$ and is also saved on the data logger.

To avoid possible biases arising from asymmetry in the column, four replicates of each experiment were conducted after a $90^{\circ}$ rotation of the column. Using different combinations of the 12 MHPM enabled from 8 to 48 replicates depending on the value of $n$, and thus a total of 116 experiments. The characteristics of the column combinations used for each value of $n$ are summarized in Table 1, as well as the number of replicates and the amount of time required by each experiment.

\section{Results}

\subsection{Experimental results}

For each value of $n$, the minimum, maximum and average breakthrough concentrations obtained from the $R$ repli-
Table 1. Experiment replicates.

\begin{tabular}{ccccc}
\hline$n$ & $V$ & $L$ & $T_{\max }$ & $R$ \\
\hline 1 & $V_{0}$ & $L_{0}$ & $15 \mathrm{~min}$ & 48 \\
2 & $2 V_{0}$ & $2 L_{0}$ & $30 \mathrm{~min}$ & 24 \\
3 & $3 V_{0}$ & $3 L_{0}$ & $40 \mathrm{~min}$ & 16 \\
4 & $4 V_{0}$ & $4 L_{0}$ & $50 \mathrm{~min}$ & 12 \\
5 & $5 V_{0}$ & $5 L_{0}$ & $60 \mathrm{~min}$ & 8 \\
6 & $6 V_{0}$ & $6 L_{0}$ & $70 \mathrm{~min}$ & 8 \\
\hline
\end{tabular}

$n$ is the number of MHPM in the column, $V$ is the pore volume $\left(V_{0}=\right.$ $461 \mathrm{ml}), L$ is the total length of the porous medium $\left(L_{0}=15 \mathrm{~cm}\right), T_{\max }$ is the total time for one experiment and $R$ the number of replicates.

cates are computed as:

$$
\begin{gathered}
C_{\min }(t)=\min _{i=1 \ldots R} C_{i}(t), \quad C_{\max }(t)=\max _{i=1 \ldots R} C_{i}(t) \\
\bar{C}(t)=\frac{1}{R} \sum_{i=1}^{R} C_{i}(t)
\end{gathered}
$$

where $C_{i}(t)$ is the output concentration for replicate $i$ at time $t$.

Figure 3 shows the experimental breakthrough curves for $n=1$ to 6 MHPM in series. For the sake of clarity, the normalized concentration is given as: $C^{*}(t)=C(t) / C_{0}$. The minimum and maximum statistical variation for the different replicates is represented by the grey envelop and the black line is the mean breakthrough curve deduced from all the replicates. It is worth mentioning the very high number of data points in comparison with other works found in the literature. This enables to draw conclusions that are not biased by a too sparse database.

Results show that for $n=1$ (only one MHPM), the breakthrough curve is far from the classical ' $S$ ' shape of a homogeneous porous medium. As expected however, the more the periodicity increases, the more we recover this shape. The behaviour for $n=1$ stems from the high contrast in porosity between the cylindrical cavity (100\%) and its surrounding medium (40\%). The salty water thus flow preferentially through this cavity, bypassing the granular medium. A consequent part of the volume flows with higher velocity and thus faster reaches the outlet. As seen in Figure 1, the fact that one of the outlets is aligned with the cylindrical cavity may enhance this behaviour.

Another important result obtained thanks to the repetition of experiments is that the breakthrough curves strongly differ from one replicate to another. The amplitude of the min- max interval seems to decrease for larger $n$, but this might be due only to the smaller number of experiments as explained in [7].

\subsection{Modelling attempt}

It is well known that the Advection-Dispersion model, based on the classical Fickian operator, may be used to recover ' $S$ '-shaped breakthrough curves obtained in homogeneous porous media. However, attempting to calibrate this model against field or laboratory data involving heterogeneous porous media has been seen to lead to contradictory conclusions (see e.g. [2, 8, 9], that may however 


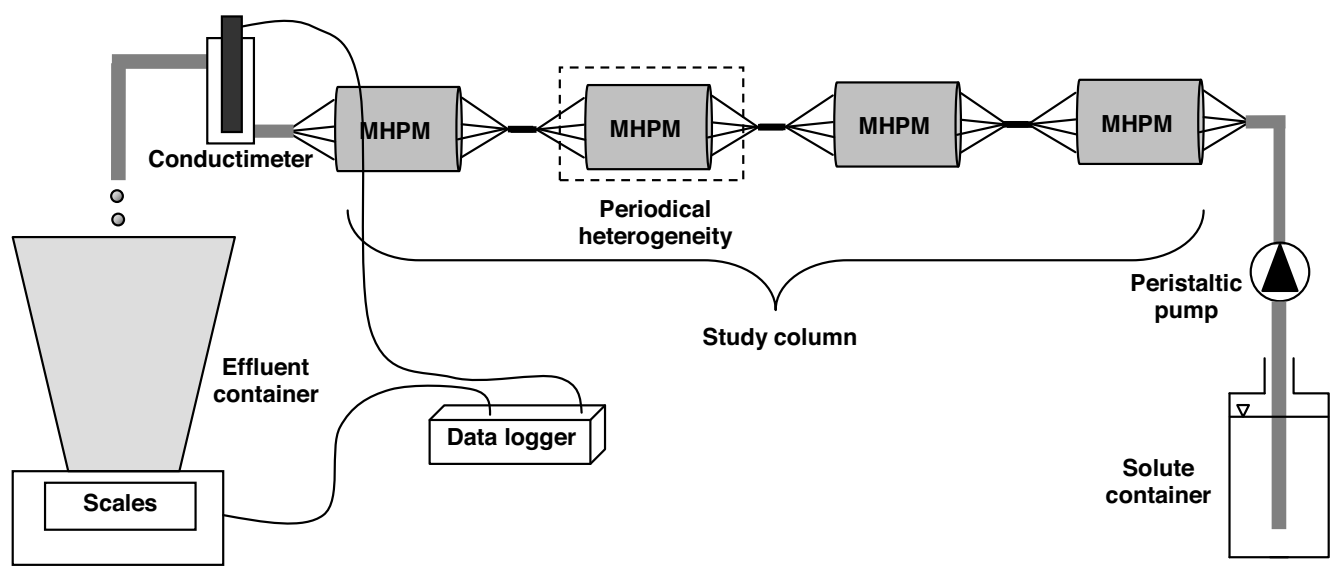

Figure 2. Experimental setup with an example of a series of $n=4$ columns.
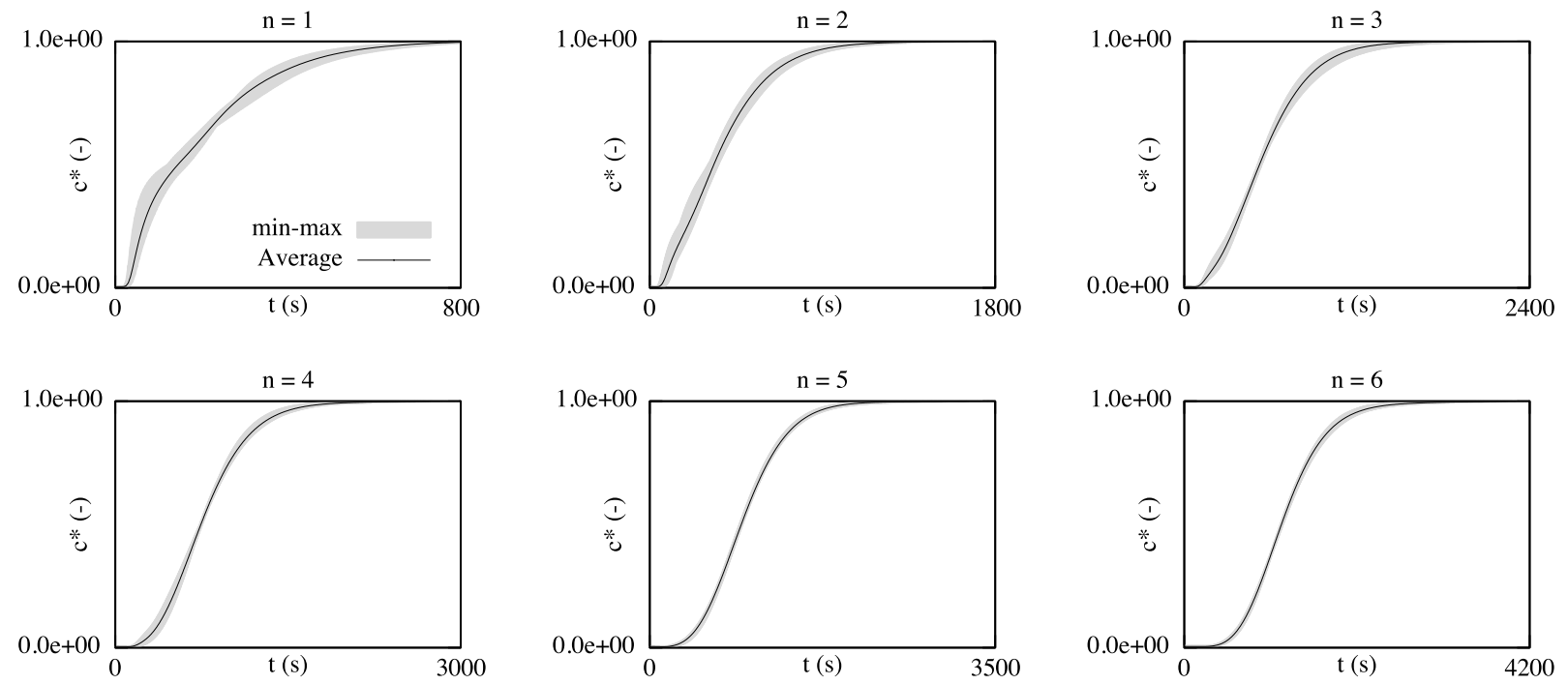

Figure 3. Experimental breakthrough curves for $n=1$ to 6 MHPM.

be tempered considering the bias induced by experimental noise.

The Advection-Dispersion model is defined as follows:

$$
\begin{gathered}
\frac{\partial C(x, t)}{\partial t}+u \frac{\partial C(x, t)}{\partial x}-D \frac{\partial^{2} C(x, t)}{\partial t^{2}}=0 \\
C(x, 0)=0 \quad \forall x \in[0, L]
\end{gathered}
$$

where $D$ is the dispersion coefficient and $u$ the flow velocity.

Using a substantial amount of experiments enabled us to show in a previous work [7] that no significant trend can be detected for the dispersion coefficient as a function of distance, given the experimental uncertainty.

In the present work, the model was calibrated in $D$ and $u$ against the six experiments ( $n=1$ to 6$)$ simultaneously. The aim being to reproduce every breakthrough curves with the same set of parameters. Result that best fits the six curves is obtained with $D=4.25 \cdot 10^{-5} \mathrm{~m}^{2} \mathrm{~s}^{-1}$ and $u=7.6 \cdot 10^{-4} \mathrm{~ms}^{-} 1$ (see Figure 4 ). As expected, this model fails to reproduce the curves for small values of $n$.

The high quality database presented in this study, will be used in forthcoming work to perform reliable calibration of several other models, that may be more prone to reproduce atypical behaviours of breakthrough curves in strongly heterogeneous porous media.

\section{Conclusions}

Our experimental results show that periodicity impacts the behaviour of solute transport in heterogeneous porous media. This highlights the importance of observation scale of solute transport. If the observation scale is larger than the heterogeneity scale, we will recover the classical ' $S$ ' shape breakthrough curve. Conversely, if the observation scale is comparable to the heterogeneity scale, the classical behaviour is lost and the breakthrough curve is no more 'S'-shaped. 

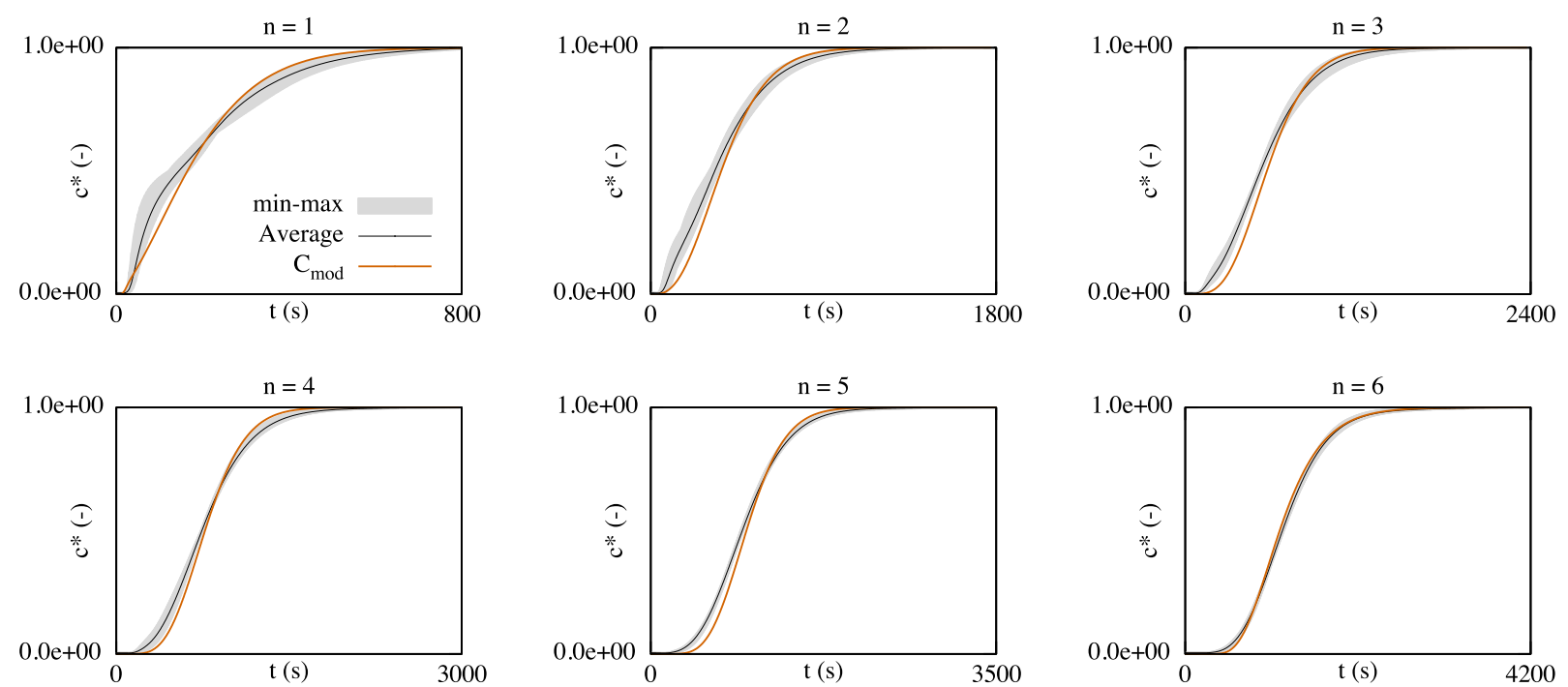

Figure 4. Modelling Results obtained with the classical Advection-Dispersion model.

An important characteristic of this work is the high number of replicates for each experiment as well as the great amount of measurements. This highlights the strong variability of the experimental results, even in the case of artificial and well controlled MHPM. Consequently, extreme caution is required when dealing with natural media.

\section{References}

[1] Silliman, S.E., Zheng, L., Conwell, P., 1998. The use of laboratory experiments for the study of conservative solute transport in heterogeneous porous media. Hydrogeol. J. 6, 166-177.

[2] Silliman, S.E., Simpson, E.S., 1987. Laboratory evidence of the scale effect in dispersion of solutes in porous media. Water Resour. Res. 23 (8), 1667?1673.

[3] Saiers, J.E., Hornberger, G.M., Hervey, C., 1994. Colloidal silica transport through structured, heterogeneous porous media. J. Hydrol. 163, 271?288.

[4] Li, L., Barry, D.A., Culligan-Hensley, P.J., Bajracharya, K., 1994. Mass transfer in soils with local stratification of hydraulic conductivity. Water Resour. Res. 30 (11), 2891-2900.

[5] Huang, K., Toride, N., Van Genuchten, M.T., 1995. Experimental investigation of solute transport in large, homogeneous and heterogeneous, saturated soil columns. Transp. Porous Media 18, 283-302.

[6] Golfier, F., Quintard, M., Woodd, B.D., 2011. Comparison of theory and experiment for solute transport in weakly heterogeneous bimodal porous media. Adv. Water Resour. 34, 899-914.

[7] Majdalani, S., Delenne, C., Chazarin J.P., Guinot, V. Solute transport in periodical heterogeneous porous media: Importance of observation scale and experimental sampling. Journal of Hydrology, 520, 52-60, 2015.

[8] Irwin, N.C., Botz, M.M., Greenkorn, R.A., 1996. Experimental investigation of characteristic length scale in periodic heterogeneous porous media. Transp. Porous Media 25, 235-246.

[9] Sternberg, S.P.K., Cushman, J., Greenkorn, R.A., 1996. Laboratory observation of nonlocal dispersion. Transp. Porous Media 23, 135-151. 